\title{
Reducing the temporal demands of the Morris place-learning task fails to ameliorate the place-learning impairment of preweanling rats
}

\author{
JERRY W. RUDY and RICHARD PAYLOR \\ University of Colorado, Boulder, Colorado
}

\begin{abstract}
Rats less than 20 days old display no evidence of place learning when trained in the Morris (1981) water task. In the present experiment, we evaluated the possibility that this impairment was a result of the immature "temporary memory buffer" that Rawlins (1985) has proposed as being critical for successful place learning. To evaluate this hypothesis, we reduced to a minimum the temporal demands of the Morris place navigation task by training pups of different ages to swim directly to a visible platform in a fixed location. All pups were then tested for place learning on the probe trial with no escape platform in the pool. In spite of being trained with a procedure that greatly reduced the temporal demands of the task, the 19-day-old pups displayed no learning of the platform's location. Several measures of place learning indicated that 22-dayolds could learn the location of the platform. Our data thus provide no support for the hypothesis that young rats are impaired on place-learning task because of an immature temporary memory buffer.
\end{abstract}

Spatial learning tasks can be divided into two categories. One class can be solved on the basis of what O'Keefe and Nadel (1978) have called taxon strategies; the other class can be solved by the use of locale strategies. The organism can learn to solve taxon problems by moving relative to a specific stimulus or by repeating the same specific movement sequence. Locale problems, however, require the organism to move directly to the goal from several different directions using the relative location of cues that may be very far away from the goal. The use of a locale strategy is sometimes referred to as "spatial mapping."

O'Keefe and Nadel (1978) have argued that taxon and locale strategies depend on neurologically distinct processes. In particular, the hippocampal formation is critical for locale strategies but not for taxon strategies. Their hypothesis has considerable support (Morris, Garrud, Rawlins, \& O'Keefe, 1982; Okaichi, 1987; O'Keefe, Nadel, Keightly, \& Kill, 1975; Sutherland, Kolb, \& Whishaw, 1982).

The findings of Morris et al. (1982) are especially relevant to our purpose. Using the Morris (1981) water task, Morris et al. found that rats with hippocampal damage performed normally when required to swim to a visible escape platform but were impaired when required to learn the location of a hidden platform. The former task required the use of only a taxon system, whereas the latter task required the use of a locale strategy.

This research was supported by National Science Foundation Grant BNS8207654. We thank R. J. Sutherland for his comments on a previous version of this article. Correspondence concerning this article should be addressed to Jerry W. Rudy, Department of Psychology, University of Colorado, Boulder, CO 80309.
Rudy, Stadler-Morris, and Albert (1987) have recently reported that the processes mediating taxon and locale forms of spatial learning are dissociated during development. Specifically, they found that rats only 17-19 days old were able to locate the visible escape platform in the Morris water task but were impaired when required to solve the hidden-platform task. On the basis of this finding and other data ruling out sensory and motivational interpretations, Rudy et al. suggested that the processes that enable the rat to learn and/or utilize the spatial mapping strategy develop late (around 20 days of age) relative to those needed to solve taxon problems (see also Dyck, Sutherland, \& Buday, 1985; Schenk, 1985). In this paper, we further clarify the difference between young and older rats on the locale version of the Morris water task.

Morris, Hagan, and Rawlins (1986) have recently challenged O'Keefe and Nadel's interpretations of the contribution of the hippocampal formation to the acquisition of spatial problems that require locale strategies. They have favored Rawlins's $(1985,1987)$ hypothesis that the hippocampal formation is a high-capacity temporary memory buffer that enables the animal to integrate temporally discontiguous events. The hippocampal formation is important in tasks that involve spatial mapping because it provides the temporary memory storage needed to construct the map.

The potential importance of such a temporary storage system to successful place learning involving an invisible platform is apparent when one considers the animal's behavior on initial trials. Typically, when the platform is invisible, the rat might take about $\mathbf{4 0} \mathrm{sec}$ to find it. Since solving the problem requires some means of integrating information acquired at the start of the trial with information experienced at the trial's end, an animal lacking 
a temporary memory buffer would be at a disadvantage in solving this task. In contrast, the visible-platform task makes no such demands on the animal's intermediate memory system.

Although one might disagree with Rawlins's view that the hippocampal formation is a temporary memory buffer, it may be true that young rats fail to solve the invisibleplatform version of the Morris water task because of the demands this task places on its immature temporary memory buffer. This hypothesis has a testable implication. If one can reduce the temporal demands of the task, one should be able to eliminate the young rat's failure to learn the spatial location of the escape platform.

Morris (1981) reported that rats will learn not only the spatial location of a hidden escape platform, but also the spatial location of a visible platform. In that case, the rat was trained to swim to a visible platform that was always in a fixed location relative to extramaze cues. The rats were then given a probe trial with the platform removed. Several measures of the rats' search strategy on the probe trial indicated that they had learned the spatial location of the platform even though they had not needed to acquire this knowledge in order to find the escape platform (see also Schenk, 1985).

This version of the Morris water task has just the properties one needs to evaluate the temporary-memorybuffer hypothesis. Within just a few trials, young rats (17-19-day-olds) swim directly to the visible platform with latencies that average less than $8 \mathrm{sec}$, thereby reducing the temporal demands this task places on the memory buffer and the amount of proactive and retroactive interference experienced. If young rats fail to learn the spatial location of the hidden platform because of an immature temporary memory buffer, then this impairment might be attenuated if they are trained with a visible platform in a fixed location relative to the extramaze cues.

\section{METHOD}

\section{Subjects}

The subjects were 16-, 19-, and 20-day-old Long-Evans-derived hooded rats bred at the University of Colorado (see Rudy \& StadlerMorris, 1987, for details of colony management). There were 6 subjects at each training age. Each of two separate litters contributed 3 subjects to each age group.

\section{Apparatus}

The training apparatus was a large circular pool, $1.2 \mathrm{~m}$ in diameter and $0.36 \mathrm{~m}$ deep. The water level in the pool was $.2 \mathrm{~m}$. Water temperature was maintained at $26^{\circ} \mathrm{C}$. The pool was located in a laboratory room that was $2.3 \times 2.73 \times 2.5 \mathrm{~m}$. The visible escape platform was a Plexiglas disk, $11.5 \mathrm{~cm}$ in diameter, with a rod extending upward from its center. A $12.5 \times 7.5 \times 5 \mathrm{~cm}$ green sponge was attached to this rod $10 \mathrm{~cm}$ above the surface of the platform. To record the subject's behavior, a television camera was mounted above the center of the pool. Other details of the room are fully described by Rudy et al. (1987). The platform was placed in the northeast quadrant of the pool with its outer edge $23 \mathrm{~cm}$ from the pool wall.

\section{Procedure}

Training. On Day 1 of training, each pup was placed on the platform for $30 \mathrm{sec}$. It was then placed in the water and allowed to swim for $30 \mathrm{sec}$. Then it was guided to the platform, where it was allowed to remain for $10 \mathrm{sec}$. After that, the subject was given 3 practice climbs onto the platform, with each climb initiated by placing the subject in the water with its forepaws against the platform. Each subject was then given its first session of 12 training trials.

Each trial was started by placing the subject (facing the wall) along the perimeter of the pool, in one of three randomly chosen quadrants of the pool. From there it was allowed $60 \mathrm{sec}$ to locate the platform and escape from the water. If it failed to locate the platform in the allotted time, it was guided onto the platform by the experimenter. Each subject received two consecutive training trials and was then returned to a holding cage until 2 other subjects had each received two trials. During the interval between the two consecutive trials, the pup remained on the platform for $35 \mathrm{sec}$. This rotation occurred among 3 animals, until all subjects had received a total of 12 trials. On separate and consecutive days the 16- and 19-day-olds received two additional training sessions, for a total of 36 trials (Groups 16-36 and 19-36); the 20-day-olds received only one additional session, for a total of 24 trials (Group 20-24). Throughout training, the visible platform remained in the same location.

Test. Testing occurred on the day following training, when the pups in Groups 16-36 and 19-36 were 19 and 21 days old, respectively. The subjects received two warm-up trials with the visible platform in the pool. Each subject then received a probe trial. On the probe trial, the platform was removed from the pool and the pup was placed into the pool at a novel starting location and allowed to search for the missing platform for $60 \mathrm{sec}$. The animal's search behavior on the probe trial was recorded on video tape for subsequent analysis.

\section{Independent Variables}

Escape latencies were recorded on each training trial. From the probe trial, five measures were obtained to characterize each subject's search pattern: quadrant-search time, number of platform crossings, heading error, path length, and peripheral pool time. To obtain the search-time measure, the pool was conceptually divided into four quadrants and the percentage of time the subject spent in each of the four quadrants was calculated. To obtain platform crossings, we counted the number of times the subject crossed the precise spot in the training quadrant where the platform had been located during training. For comparison, the number of times the subject crossed the equivalent location in each of the other three quadrants was also recorded. Heading error was obtained by noting the direction in which the pup's head was pointed once it was $15 \mathrm{~cm}$ from the wall. The distance (in degrees) that this path would take the pup from the platform was then calculated. A score of $0^{\circ}$ indicates that the path would lead directly to the platform. Path length was obtained by measuring the distance (in centimeters) the animal swam from the time it was released until it made its first platform crossing. Peripheral pool time was measured by dividing the pool into two concentric circles-a center circle with a radius of $37 \mathrm{~cm}$ and an outer ring width of $23 \mathrm{~cm}$, as measured from the inside wall of the tank-and calculating the time the pup spent in the center circle and in the outer ring. It should be noted that the platform was located within the center ring.

\section{RESULTS}

\section{Acquisition}

The acquisition data are presented in Figure 1. Two points are noteworthy. First, when the 16-day-olds are compared with the 19-day-olds (Group 16-36 vs. Group 19-36), a clear difference is observed in their escape latencies $[F(1,10)=22.5, p<.01]$. This differ- 


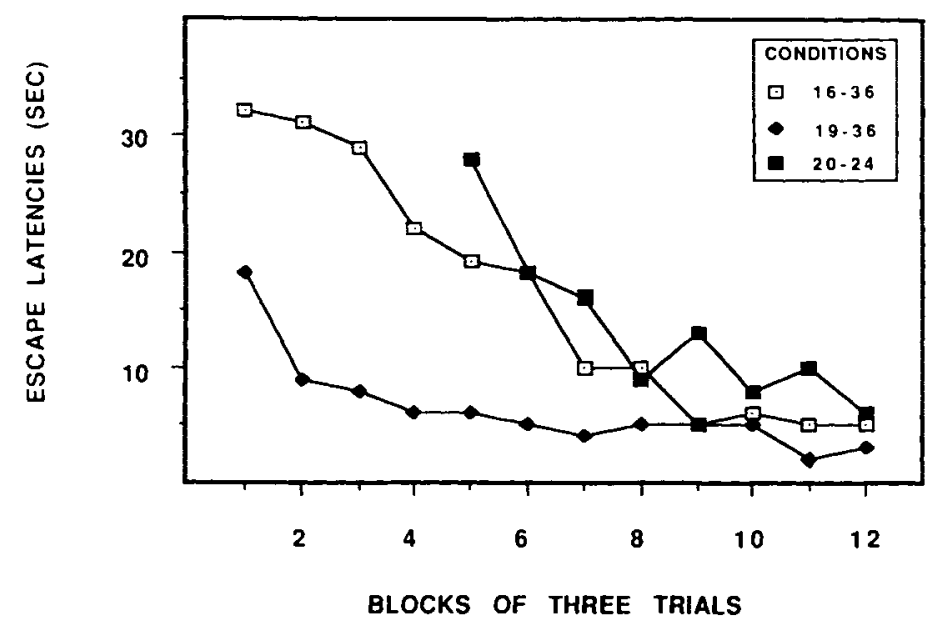

Figure 1. The mean escape latencies for the three groups during training with the visible platform. Note that the subjects in Group 20-24 received only 24 trials, whereas the subjects in Groups 16-36 and 19-36 received 36 trials. Note also that the data for Group 20-24 are plotted so that they correspond to the last 24 trials of training for Groups 16-36 and 19-36.

ence is potentially important to the purposes of the experiment, because an adequate test of the temporarymemory-buffer hypothesis requires that the different-aged pups receive not only the same number of trials, but also the same number of functionally equivalent trials, trials with equivalently short latencies. This requirement could not be fulfilled, because the escape latencies of the 19day-olds were shorter on the initial two training sessions (Trial Blocks 1-8 in Figure 1). Second, however, note that the 16-day-olds' performance over their last 24 training trials was equivalent to the 20-day-olds' performance over their 24 training trials (compare Group 16-36 and 20-24 over Trial Blocks 5-12 in Figure 1). Thus, although the test data comparing Groups $16-36$ and $19-36$ is complicated by the fact that the 19-day-olds had significantly shorter escape latencies, the comparison of Groups $16-36$ and $20-24$ is not.

\section{Test Data}

The results of the probe trial are easily summarized. the 16-day-olds displayed no evidence of learning the spatial location of the platform, even though they were able to swim directly to the visible platform. In contrast, all measures of search behavior converge to indicate that both sets of older animals learned the location of the platform.

Figure 2 presents the quadrant search times and platform crossings for the three sets of animals. Note that the pups in Group 16-36 did not selectively search the training quadrant, and that their platform crossings were not selectively constrained to the training quadrant $[F \mathrm{~s}(3,15)<1.50]$. In contrast, the older pups, whether they had received only 24 trials (Group 20-24) or 36 trials (Group 19-36), did selectively search the training quadrant $[F \mathrm{~s}(3,15)>3.7, p<.05]$ and made more platform crossings in that quadrant $[F \mathrm{~s}(3,15)>5.0, p<.01]$ than in the other three quadrants. Post hoc comparisons of the training-quadrant search time and platform-crossing scores of Groups 19-36 and 20-24 with their scores in each other quadrant were all reliable $(p<.05$, Dunnett test), with the one exception that Group 19-36's trainingquadrant search time was not reliably different $(p<.10)$ from its search time in one adjacent quadrant.

The mean heading errors were $53^{\circ}, 18^{\circ}$, and $26^{\circ}$ for the Groups $16-36,19-36$, and $20-24$, respectively. They indicate that the initial trajectories of the older animals, which did not differ, were significantly more accurate than the trajectories of the younger pups $[F(2,15)=4.3$, $p<.05$ ].

The mean path lengths were 87,26 , and $43 \mathrm{~cm}$ for Groups 16-36, 19-36, and 20-24, respectively. The analysis of variance on these data failed to approach significance $[F(2,15)=2.00, p>.10]$. However, a comparison of the average path length of Group 16-36 $(87 \mathrm{~cm})$ with the average path length for the older animals in Groups 19-36 and 20-24 combined (34 cm) indicated that the older pups' path lengths were reliably shorter than the younger pups' $[t(16)=1.98, p<.05$, one-tailed test].

The peripheral-pool-time measure did not differentiate the young and old pups. The mean times spent in the center circle were 16,18 , and $16.8 \mathrm{sec}$ and the mean times spent in the outer ring were 44,42 , and $43.8 \mathrm{sec}$ for the three conditions. These results indicate that the young animals spent no more time in the perimeter of the pool than did the older animals.

\section{DISCUSSION}

These findings offer no support for the idea that young rats perform poorly on spatial mapping tasks because of an immature temporary memory buffer. Even though the young pups were trained under conditions that should 

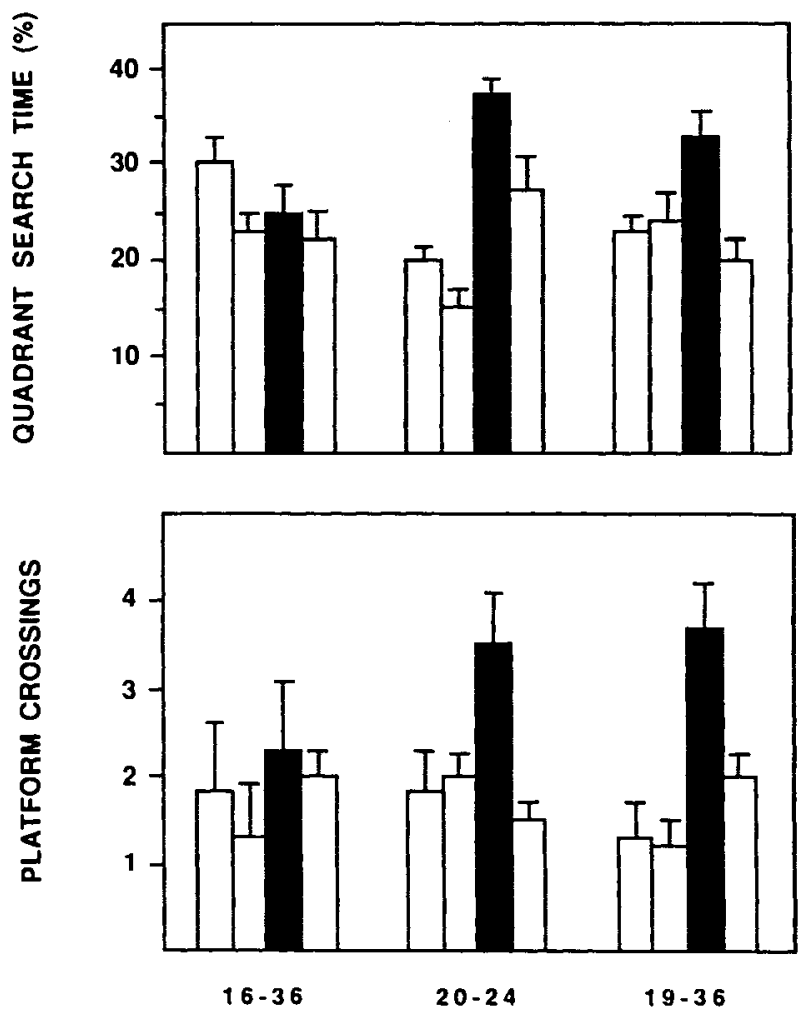

CONDITIONS

Figure 2. The top and bottom panels present, respectively, the mean quadrant search times and the mean number of platform crossings for each group on the probe trial. Note that the subjects in Groups 19-36 and 20-24 each selectively searched the training quadrant (represented by the solid bar) more, and crossed its platform location more often, than they did the other quadrants. In contrast, the youngest pups (Group 16-30) did not engage in selective search behavior on the probe trial.

minimize, as much as possible, the demands that the Morris water task places on a temporary memory buffer, 16-19-day-olds failed to demonstrate any evidence of having learned the location of the platform.

These data also do not support another interpretation of why the 17-19-day-old rats in the Rudy et al. (1987) experiments failed to display evidence of place learning. When their 18-19-day-old rats were trained to find a hidden platform in a fixed location, their escape latencies were very long, averaging about $50 \mathrm{sec}$ (see Rudy et al., 1987, Figure 3). Apart from any demands that such latencies place on the animal's temporary memory buffer, one has to worry that these young pups might be so fatigued by the end of a trial that the processes subserving place learning were impaired. In the present experiment, the escape latencies of the 16-19-day-olds were much shorter, especially over the last training session, in which their average escape latency was about $5 \mathrm{sec}$. This suggests that the presence of the visible platform greatly reduced the effects fatigue could have on the place learning of the youngest animals. Nevertheless, they failed to learn the location of the platform.

Although 16-19-day-olds do not utilize the locale strategy, it is obvious that they can readily utilize a taxon strategy, that is, approach a visible object. The present data thus support Rudy et al.'s (1987) original conclusion that the processes mediating spatial mapping strategies and those mediating taxon strategies are dissociated during ontogenesis. Neither the present experiment nor those of Rudy et al. (1987) provide any evidence that rats use locale strategies until they are about 20 days old, whereas they can use a taxon strategy when they are only 16-17 days old. Since the rat's eyes do not open until about Day 15 after birth, this means that the processes underlying the use of visually mediated taxon strategies may be ready to function when they first receive a discriminable visual input.

The integrity of the hippocampal formation is critical if the animal is to solve spatial problems that require the use of locale strategies (Okaichi, 1987; O'Keefe et al., 1975; Morris et al., 1982; Sutherland et al., 1982; Sutherland, Whishaw, \& Kolb, 1983). It is thus of interest to note that the hippocampal formation matures substantially during the period between eye opening and when the animal can learn the spatial location of the escape platform in the Morris water task (Altman \& Das, 1965; Cotman, Taylor, \& Lynch, 1973; Crain, Cotman, Taylor, \& Lynch, 1973; Meibach, Ross, Cox, \& Glick, 1981; Pokorny \& Yamamoto, 1981).

To the degree that maturational differences in the hippocampal formation contribute to the age-related differences in spatial location learning that Rudy et al. (1987) and we found, our data provide no support for Rawlins's (1985, 1987; Morris et al., 1986) view of the hippocampal formation as a temporary intermediate memory buffer. Contrary to the implications of Rawlins's view, reducing the interval within a trial over which spatial information must be integrated did not improve the young rat's ability to learn the location of the platform. Even so, it is not clear that the present experiment should be considered to be a critical test of Rawlins's theory. First, we do not know with any certainty that it is maturation of the hippocampal formation that is crucial to the age-related effects we have reported. Second, one could argue that the $5 \mathrm{sec}$ taken by the animals to complete a trial was still too long to be bridged by animals lacking hippocampal function. If this argument is correct, however, it is not clear why animals with complete hippocampectomies can bridge 60 -sec delays in a pseudotrial unique recognitionmemory task (Aggleton, Hunt, \& Rawlins, 1986).

\section{REFERENCES}

Aggleton, J. P., Hunt, P. R., \& Rawlins, J. N. P. (1986). The effects of hippocampal lesions upon spatial and non-spatial tests of working memory. Behavioral Brain Research, 19, 133-146. 
Altman, J., \& Das, G. D. (1965). Audiographic and histological evidence of postnatal neurogenesis in rats. Journal of Comparative Neurology, 124, 319-336.

Cotman, C., TAYlor, D., \& LYNCH, G. (1973). Ultrastructural changes in synapses in the dentate gyrus of the rat. Brain Research, 63, 205-213.

Crain, B., Cotman, C., Taylor, D., \& Lynch, G. (1973). A quantitative electron microscopic study of synaptogenesis in the dentate gyrus of the rat. Brain Research, 63, 195-204.

Dyck, R. H., Sutherland, R. J., \& Buday, M. R. (1985). The ontogeny of mapping and nonmapping spatial strategies following neonatal hippocampal damage in rats. Society for Neuroscience $A b$ stracts, 11,832 .

Meibach, R. C., Ross, D. A., Cox, R. D., \& Glick, S. D. (1981). The ontogeny of hippocampal energy metabolism. Brain Research, 204, $431-435$.

MorRIS, R. G. M. (1981). Spatial location does not require the presence of local cues. Learning \& Motivation, 12, 239-260.

Morris, R. G. M., Garrud, P., Rawlins, J. N. P., \& O'Keefe, J. (1982). Place navigation impaired in rats with hippocampal lesions. Nature, 297, 681-683.

Morris, R. G. M., Hagan, J. J., Rawlins, J. N. P. (1986). Allocentric spatial learning by hippocampectomised rats: A further test of the "spatial mapping" and "working memory" theories of hippocampal function. Quarterly Journal of Experimental Psychology, 38B, 365-395.

OKaICHI, H. (1987). Performance and dominant strategies on place and cue tasks following hippocampal lesions in rats. Psychobiology, 15, 58-63.

OKEEFE, J., \& NADEL, L. (1978). The hippocampus as a cognitive map. Oxford: Clarendon Press.
O'Keefe, J., Nadel, L., Keightly, S., \& Kill, D. (1975), Fornix lesions selectively abolish place learning in the rat. Experimental Neurology, 48, 152-166.

Pokorny, J., \& Yamamoto, T. (1981). Postnatal ontogenesis of hippocampal CAl area in rats: I. Development of dendritic arborization in pyramidal neurons. II. Development of ultrastructure in stratum lacunosum and moleculare. Brain Research Bulletin, 7, 113-130.

Rawlins, J. N. P. (1985). Associations across time: The hippocampus as a temporary memory store. Behavioral \& Brain Sciences, 8 , $479-496$

Rawlins, J. N. P. (1987). Time to close the store? Behavioral \& Brain Sciences, 10, 156-159.

RudY, J. W., \& SADler-Morris, S. (1987). The development of interocular transfer by rats trained on a distal-cue navigation task. Behavioral Neuroscience, 101, 141-143.

Rudy, J. W., Stadler-Morris, S., \& Albert, P. (1987). Ontogeny of spatial navigation behaviors in the rat: Dissociation of "proximal" and "distal" cue-based behaviors. Behavioral Neuroscience, 101, $62-73$.

SCHENK, F. (1985). Development of place navigation in rats from weaning to puberty. Behavioral \& Neural Biology, 43, 69-85.

Sutherland, R. J., Kolb, B., \& Whishaw, 1. Q. (1982). Spatial mapping: Definitive disruption by hippocampal or medial frontal damage in the rat. Neuroscience Letters, 31, 271-276.

Sutherland, R. J., Whishaw, I. Q., \& KolB, B. (1983). A behavioral analysis of spatial localization following electrolytic, kainate-, or colchicine-induced damage to the hippocampal formation in the rat. Behavioral Brain Research, 7, 133-153.

(Manuscript received October 4, 1987; revision accepted for publication February 27,1988 .)

\section{ERRATA}

Two errors involving authors' names occurred in two recent issues of Psychobiology. We very much regret these errors, and apologize for any confusion they may have caused. They are the following:

Wilson, B., Kolb, B., Odland, L., \& Whishaw, I. Q. Alcohol, sex, age, and the hippocampus (Psychobiology, 1987, 15, 300-307)-I. Q. Whishaw's name was misspelled in the author line of the article. The correct spelling is as given here. (I. Q. Whishaw's name was also misspelled in the list of reviewers on page $v$ of the same issue.)

Jacobs, W. J., Blackburn, J. R., Buttrick, M., Harpur, T. J., Kennedy, D., Mana, M. J., MacDonald, M. A., McPherson, L. M., Paul, D., \& Pfaus, J. G. Observations (Psychobiology, $1988,16,3-19)-W$. J. Jacobs's name was omitted from the table of contents on the cover of the issue. 